\title{
Dynamics and Removal Pathway of Edge Dislocations in Imperfectly Attached Nanocrystal Pairs; Towards Design Rules for Oriented Attachment
}

Justin Ondry $^{1}$, Matthew R. Hauwiller ${ }^{1}$ and A. Paul Alivisatos ${ }^{1,2,3,4}$

1. Department of Chemistry, University of California, Berkeley, California, United States.

2. Department of Materials Science and Engineering, University of California Berkeley, Berkeley, California, United States.

3. Materials Sciences Division, Lawrence Berkeley National Laboratory, Berkeley, California, United States

4. Kavli Energy NanoScience Institute, University of California Berkeley and Lawrence Berkeley National Laboratory, Berkeley, California, United States

Oriented attachment of nanocrystals is a common crystal growth process in biological, geologic, and synthetic nanomaterials.[1] Recently, some beautiful schemes for synthesizing epitaxially connected nanocrystal superlattices through oriented attachment have been developed.[2] These structures are theorized to have exotic electronic structures such as Dirac cones and non-trivial flat bands, but these properties have yet to be realized. Defects formed during nanocrystal attachment may be the culprit.

When nanocrystals with surface step edges epitaxially attach, it can lead to the formation of a dislocation in the nanocrystal. [3] From an electronic materials perspective, it would be desirable to understand how to remove these defects since they can have deleterious effects. Here we show through HRTEM that imperfect oriented attachment of nanocrystals creates well-defined dislocations which can be described by a burgers vector and well-established dislocation theory. We show that for PbTe nanocrystals, attachment happens on both $\{100\}$ and $\{110\}$ facets (Figure 1A-D). In both cases, $\mathrm{b}=\mathrm{a} / 2\langle 110\rangle$ edge dislocations result which are glissile dislocations in the rock salt system. From the burgers vector, we can determine the glide plane of the dislocations, and in the case of $\{100\}$ attachment, the glide plane quickly intersects the surface leading to easy defect removal (Figure 1E). In the $\{110\}$ attachment case we again observe $b=a / 2<110\rangle$ edge dislocations, but in this case the glide plane is colinear with the attachment direction. We observe that this dislocation is much more difficult to remove under the same electron dose rate (Figure 1F). From this, we determine that for $\mathrm{PbX}$ nanocrystals, attachment on the $\{100\}$ facets is superior because, if a dislocation is formed, it easily follows it's glide plane to the surface where it is annihilated. As a more general design rule, the glide plane of a possible dislocation must intersect a surface at a large angle for defect-free oriented attachment of nanocrystals.

Another important class of nanomaterials are the II-VI nanocrystals (CdSe, CdS, etc.) which have the tetrahedral zinc blende or wurtzite structure. In this case, the dislocations and glide planes that may result are distinct from the rock salt structure and the ideal attachment facets may be different. To begin investigating these structures we have induced attachment on $\{1100\}$ facets of wurtzite CdSe/CdS platelike structures (Figure 2A, B). In this case, we observe the formation of $b=\langle 1210\rangle$ type edge dislocations (Figure 2C). In this geometry, the glide plane of the dislocations is such that they would have to travel across the entire nanocrystal before being removed and is likely an unfavourable attachment direction. The lower symmetry of the tetrahedral semiconductors means there are several unique attachment directions and thus dislocation geometries that can form, meaning further investigation is required. We have thus shown that well-established dislocation theory can provide guidelines for preparing defect free nanocrystal assemblies. 


\section{References:}

[1] J. J. De Yoreo et al., Science, 349,aaa6760-1-aaa6760-9(2015).

[2] M. P. Boneschanscher et al., Science. 344, 1377-1380 (2014).

[3] R. L. Penn, J. F. Banfield, Science. 281, 969-971 (1998).

[4] The work was supported by the U.S. Department of Energy, Office of Science, Office of Basic Energy Sciences, Materials Sciences and Engineering Division, under Contract No. DE-AC02-05-CH11231 within the Physical Chemistry of Inorganic Nanostructures Program (KC3103).

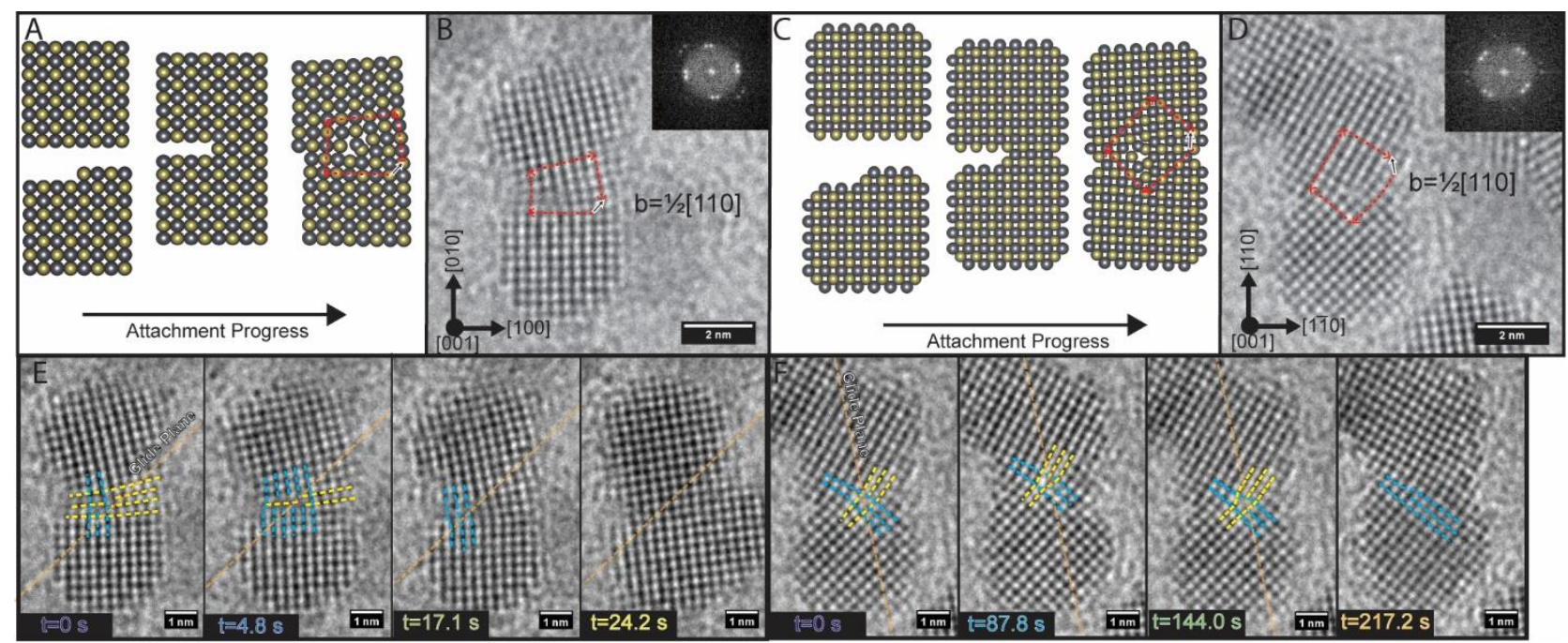

Figure 1: (A) and (B) Model and HRTEM image showing imperfect attachment of PbTe nanocrystals on $\{100\}$ facets resulting in $\mathrm{a} b=\mathrm{a} / 2\langle 110\rangle$ edge dislocation. (C) and (D) Model and HRTEM image showing imperfect attachment of PbTe nanocrystals on $\{110\}$ facets resulting in $a b=a / 2<110>$ edge dislocation. (E) HRTEM movie of $\{100\}$ imperfectly attached nanocrystals where the defect easily follows the glide plane to the surface. (F) HRTEM movie of $\{110\}$ attached nanocrystals, where defect removal is difficult due to the co-linear glide plane.

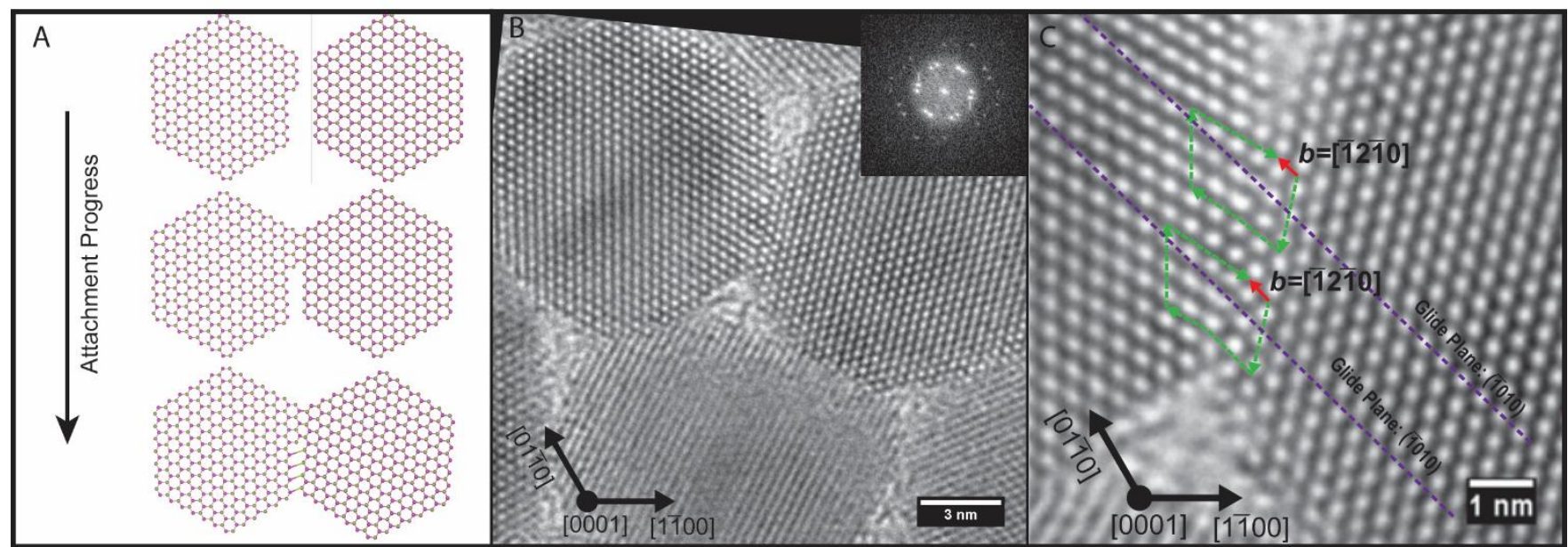

Figure 2: (A) Process which will lead to imperfect oriented attachment on $\{1100\}$ planes. (B) HRTEM image of CdSe/CdS plate-like nanocrystals viewed down the [0001] zone axis. (C) zoomed in view of the attachment interface showing that the dislocations formed have $b=[\overline{1} 2 \overline{1} 0]$ burgers vector. 